\title{
CONTINUOUS AND PROPER DECOMPOSITIONS
}

\author{
G. K. WILLIAMS ${ }^{1}$
}

Abstract. If $X$ is a locally connected, locally peripherally compact Hausdorff space and if $R$ is an equivalence relation on $X$ with fibers which are connected with compact boundaries, then it is shown that three definitions of continuity of $R$ are equivalent. Some of the propositions used to obtain this result are then applied to get sufficient conditions for a decomposition of certain types of metric spaces to be proper.

1. Introduction. The notion of continuity for decompositions of topological spaces goes back to R. L. Moore [6] and Alexandroff [1]. In recent years this concept and that of a proper decomposition have been quite useful in analysis as will be noted, for example, in [2], [4], [5], [7]. There are several definitions of continuity which are, in general, not equivalent and one purpose of this note is to give conditions under which they are equivalent. We also make some observations concerning proper decompositions. Essential use is made of the results and methods in [3] and [9].

2. Definitions and terminology. Consider an equivalence relation $R$ on a topological space $X$. Given a set $S \subset X, R(S)$ is the set of all points $x \in X$ such that $x$ is equivalent to some point of $S$ and is called the saturation of $S$. In particular, if $x \in X$, then $R(x)$ is called a fiber. If $F$ is a filterbase on $X$ then $R$ generates a filterbase, $F_{R}$, each element of which is the saturation of some element of $F$. The set of accumulation points of a filterbase $F$ will be denoted by $A(F)$.

A relation $R$ is open (closed) if the saturation of every open (closed) set in $X$ is open (closed). $R$ is Hausdorff if the quotient space is Hausdorff. $R$ is proper if the saturation of every compact set is compact.

3. Continuity conditions. Let $X, R$ be given and let $p: X \rightarrow X / R$ be the natural projection. Then consider the conditions:

I. $R$ is open.

II. $R$ is closed.

Received by the editors November 3, 1969.

AMS 1969 subject classifications. Primary 5422, 5425.

Key words and phrases. Semicontinuous decompositions, continuous decompositions, proper decompositions, open equivalence relation, closed equivalence relation, semiclosed equivalence relation, locally peripherally compact space.

1 This work was partially supported by an NSF COSIP grant. 
III. For every filterbase $F$ converging to $x \in X, R(x) \subset A\left(F_{R}\right)$.

IV. For every filterbase $F$ converging to $x \in X, A\left(F_{R}\right) \subset R(x)$.

If $R$ satisfies I (II) it is called lower (upper) semicontinuous in the open set sense. Condition II is obviously equivalent to:

II'. For every open set $U \subset X$ the union of all fibers contained in $U$ is open.

If $R$ satisfies III (IV) it is called lower (upper) semicontinuous in the limit sense.

Consider now the conditions:

$\mathrm{C}_{1}: R$ satisfies I and II.

$\mathrm{C}_{2}: R$ satisfies III and IV.

$\mathrm{C}_{3}$ : (1) $R$ is open, (2) $R$ is semiclosed, i.e., the saturation of every compact set is closed and (3) if $U \subset X / R$ is an open connected set then each component of $p^{-1}(U)$ maps on to $U$.

$\mathrm{C}_{1}$ is continuity in the open set sense, $\mathrm{C}_{2}$ is continuity in the limit sense and $C_{3}$ was proposed by Whyburn and studied in [9] and [10].

It is well known (see [3] or [4]) that I and III are equivalent conditions and that $\mathrm{C}_{2}$ holds if and only if $R$ is open and Hausdorff. For first countable spaces $C_{2}$ is equivalent to: for every sequence $\left\{x_{n}\right\}$, $\left\{x_{n}\right\} \rightarrow x$ implies $R\left(x_{n}\right) \rightarrow R(x)$. Also, for first countable spaces condition IV is equivalent to:

IV'. Any fiber which intersects the limit inferior of a sequence of fibers contains the limit superior of this sequence.

Using the above remarks and [9, Theorem 1.1] we have the following result.

Proposition 1. If $X$ is first countable and $R$ is open and semiclosed, then $R$ is $\mathrm{C}_{2}$.

As a corollary to Proposition 1 we get the well known fact that if $X$ is a first countable space in which compact subsets are closed, then $X$ is Hausdorff.

Proposition 2. If $X$ is a locally connected Hausdorff space and $R$ is $\mathrm{C}_{1}$, then $R$ is $\mathrm{C}_{3}$.

Proof. Since $X$ is Hausdorff and $R$ is closed, the saturation of every compact set is closed.

Suppose $U$ is some region in $X / R$ and $Q$ is a component of $p^{-1}(U)$ which does not map onto $U$. Then there is a point $y \in U$ which is a limit point of $p(Q)$ with $y \in U-p(Q)$. Let $V \subset X$ be the union of the remaining components of $p^{-1}(U) . V$ is then open and contains $p^{-1}(y)$ and hence $V$ contains an open saturated set $V^{\prime}$ which contains $p^{-1}(y)$. Thus $p\left(V^{\prime}\right) \subset U$ is an open set which contains $y$ but $p\left(V^{\prime}\right) \cap p(Q)=\varnothing$. 
The proof of the following proposition is similar to that of [9, Theorem 7.1] and will be omitted.

Proposition 3. Let $X$ be a locally connected, locally peripherally compact Hausdorff space and suppose $R$ is $C_{3}$ with fibers which have compact boundaries. Then $R$ is closed and hence $\mathrm{C}_{1}$.

We can now give conditions which imply the equivalence of the three continuity definitions.

THEOREM. If $X$ is a locally connected, locally peripherally compact Hausdorff space and the fibers of $R$ are connected with compact boundaries, then $\mathrm{C}_{1}, \mathrm{C}_{2}$, and $\mathrm{C}_{3}$ are equivalent.

Proof. The equivalence of $\mathrm{C}_{1}$ and $\mathrm{C}_{2}$ is proved in [3, Satz 7] and the equivalence of $C_{1}$ and $C_{3}$ follows from Propositions 2 and 3.

The connectedness is necessary as is shown by the following wellknown example.

EXAMPLE. Let $S \subset E^{2}$ be the set

$$
S=\{(x, y) \mid y=0,0 \leqq x \leqq 1\} \cup\{(x, y) \mid y=x, 0 \leqq x<1 / 2\} .
$$

Two points are $R$ equivalent if they lie on the same vertical line. Then $R$ is $\mathrm{C}_{2}$ with compact fibers but not $\mathrm{C}_{1}$ or $\mathrm{C}_{3}$.

The point-inverse decomposition of the complex exponential function is an example which is $\mathrm{C}_{2}$ and $\mathrm{C}_{3}$ but not $\mathrm{C}_{1}$.

4. Proper decompositions. If $R$ is a closed equivalence relation with compact fibers, then $R$ is proper. We also note that a fiber of an open and semiclosed relation is either an open set or is its own boundary.

Proposition 4. If $X$ is connected, locally peripherally compact and metric and if $R$ (nondegenerate) is open and semiclosed with fibers which are connected with compact boundaries, then $R$ is proper.

Proof. Since $R$ is open and semiclosed and $X$ is connected it is easy to see that the fibers of $R$ are compact. That $R$ is closed follows from Proposition 1 and [3, Satz 7].

Proposition 5. If $X$ is connected, locally connected, locally peripherally compact and metric and if $R$ (nondegenerate) is $\mathrm{C}_{\mathbf{3}}$ with fibers which have compact boundaries, then $R$ is proper.

Proof. The proof is similar to that of Proposition 4 except that here we use Proposition 3.

In both cases $X / R$ is metric by the results of [8]. 


\section{REFERENCES}

1. P. Alexandroff and H. Hopf, Topologie. Vol. I, Springer, Berlin, 1935.

2. H. Cartan, Quotients of analytic spaces, Internat. Colloq. Contribution to Function Theory (Bombay, 1960), Tata Institute of Fundamental Research, Bombay, 1960, pp. 1-15. MR 25 \#3199.

3. J. Flachsmeyer, Über halbstetige Zerlegungen topologischer Räume, Math. Nachr. 24 (1962), 1-12. MR 26 \#5534.

4. H. Holmann, Komplexe Räume mit komplexen Transformationsgruppen, Math. Ann. 150 (1963), 327-360. MR 27 \#776.

5. I. Lieb, Über komplexe Räume und komplexe Spektren, Invent. Math. 1 (1966), 45-58. MR 33 \#5939.

6. R. L. Moore, Foundations of point set theory, Amer. Math. Soc. Colloq. Publ., vol. 13, Amer. Math. Soc., Providence, R. I., 1932.

7. K. Stein, Analytische Zerlegung komplexer Räume, Math. Ann. 132 (1956), 63-93. MR 18, 649.

8. A. H. Stone, Metrizability of decomposition spaces, Proc. Amer. Math. Soc. 7 (1956), 690-700. MR 19, 299.

9. G. T. Whyburn, Continuous decompositions, Amer. J. Math. 71 (1949), 218226. MR 10, 317.

10. - Open mappings on locally compact spaces, Mem. Amer. Math. Soc. No. 1 (1950). MR 13, 764.

Southwestern at Memphis, Memphis, Tennessee 38112 